\title{
Fat rats skew research results
}

Failure to recognize that many laboratory animals live unhealthy lives may be leading researchers to misinterpret their findings, potentially misdirecting efforts to develop theraputic drugs.

The problem, reports a group at the US National Institute on Aging in Bethesda, Maryland, is that many rats and mice used in experiments are so overweight that they are glucose intolerant and heading for an early death (B. Martin et al. Proc. Natl Acad. Sci. USA doi:10.1073/pnas.0912955107; 2010). As a result, data from the animals - about, for example, the effects of an anti-cancer drug - may not apply to normal-weight animals.

"The vast majority of investigators who use rats and mice don't recognize that their normal conditions are relatively unhealthy," says Mark Mattson, chief of the National Institute on Aging's Laboratory of Neurosciences and a co-author on the paper. "The most logical way to extrapolate is to say any data we obtain in the animal model would be more relevant to overweight, sedentary humans than normalweight, active individuals."

Mattson and his colleagues note that the standard lab practice of allowing rats and mice continuous access to food without much opportunity to exercise can cause some to balloon in weight to up to 1 kilogram. Beneficial effects of a potential drug or behaviour could simply result from its effect on the consequences of an animal's unhealthy lifestyle, they say, and studies showing that caloric restriction can extend lifespan may have to be reinterpreted. "A major reason the lifespan of rats and mice is extended by caloric restriction is they started from an unhealthy baseline," argues Mattson. He and his co-workers identify areas as diverse as immune function, cancer and neurological disorders that could be affected by the problem.

Mattson says that including running wheels in cages and feeding only on alternate days could solve the 'fat rat' problem, adding that the institutional committees that oversee and approve such experiments should encourage researchers to tackle the problem.

The fat-rat hypothesis is certainly credible, says Robin Franklin, a neuroscientist and director of research at the Department of Veterinary Medicine, University of Cambridge, UK. "But I suspect it's one of many factors that are responsible for the difference between animal models and human diseases," he says, adding that the problem has not been apparent in his research.

Still, Mattson and his colleagues have penned "hundreds of papers using rodent models and are widely known in their field", says Christian Newcomer, executive director of the Association for Assessment and Accreditation of Laboratory Animal Care, a non-profit organization based in Frederick, Maryland, that accredits animal use in many laboratories, including those of the US National Institutes of Health. "I think [the paper] is going to carry a lot of weight."

Daniel Cressey 